\title{
Triplex solution for a monogenic problem
}

\section{By Lauren Martz, Staff Writer}

Researchers at Yale University School of Medicine have designed triplex-forming peptide nucleic acids for the targeted genetic correction of monogenic blood disorders, a method which they propose could be safer and simpler than conventional approaches such as gene therapy.

The group showed that their peptide nucleic acids (PNAs) corrected mutations in the human hemoglobin- $\beta(H B B)$ gene in a site-specific fashion, ${ }^{1}$ and a company has already picked up the technology. But other academics and companies contacted by SciBX think the frequency of genetic correction needs improvement before it can be considered therapeutically useful.

Mutations in the second intron of the $H B B$ gene cause thalassemia, a blood disorder that leads to a deficiency in hemoglobin. ${ }^{2}$ The current treatment involves regular blood transfusions and the use of iron-chelating agents to limit iron overload from the transfusions. How-

"The length of the target and of the PNA must be increased to guarantee a specific effect and limit offtarget effects."

-Carine Giovannangeli, Museum of Natural History

disrupted with chloroquine post-transfection.

In the $\mathrm{S}$ phase, the opened chromatin structure of the native DNA likely made the binding site for the PNAs more accessible. PNAs also tend to be taken up by the lysosomes, and, as a result, lysosome disruption is necessary for PNAs to exert their effect.

\section{The ex factor}

All told, Glazer said the paper shows that PNAs can promote targeted genetic correction ex vivo. He proposes that subsequent transplantation of the corrected cells back into patients might provide a therapeutic benefit.

"The idea is to take the hematopoietic stem cells from individuals with sickle cell disease, thalassemia or other blood diseases through a clinically practiced and minimally invasive procedure that involves mobilizing the stem cells to the peripheral blood using intravenous growth factors," Glazer said. "The stem cells are treated with the PNA molecules ex vivo. Electroporation treatment is used to deliver the PNAs and the donor DNA into the cells. Once the cells have recovered, they can be intravenously infused back into the blood and will have a tendency to migrate back to the bone marrow where they will make normal hemoglobin."

He added that the strategy could allow for long-term treatment because the corrected stem cells give rise to blood cells of both erythroid and myeloid lineage. Most importantly, in the reported research, both of those cell types had the corrected $H B B$ gene.

"The paper introduces a very strong and interesting approach," said C. Frank Bennett, SVP of research at RNA company Isis Pharmaceuticals Inc.

ever, therapeutic success is highly dependent on

patient compliance, and chelating agents can cause side effects such as hepatic dysfunction and cytopenias.,

PNAs contain a peptide-like backbone and the standard bases, purines and pyrimidines. The oligomers bind to DNA at specific sites by forming triplexes with complementary single-stranded DNA. Previous studies showed that PNAs promote genetic recombination and repair at sites in close proximity to the PNA binding site on plasmid DNA. ${ }^{5}$

Now, Peter Glazer and colleagues have reported in the Proceedings of the National Academy of Sciences the development of PNAs that promote genetic recombination in cells, including in hematopoietic stem cells. Glazer is chairman of the Department of Therapeutic Radiology and professor of genetics at the medical school.

The researchers transfected either human erythroleukemic K562 cells, mouse bone marrow cells containing human $H B B$ or human CD $34^{+}$hematopoietic progenitor cells using PNAs and donor oligonucleotides containing the correct $H B B$ sequence. The PNAs bound at sites on the endogenous DNA ranging from 35-830 base pairs from the $H B B$ mutation site and elicited gene correction.

The highest rates of genetic correction occurred when the cells were in the $\mathrm{S}$ phase of the cell cycle or when the cells' lysosomes were
He said an ex vivo approach is optimal because PNAs have poor pharmacokinetics in vivo. The compounds are relatively insoluble due to their neutral amino-like backbone, he said.

"The pharmacokinetic problem with insoluble PNAs is that they might be excreted through the urine very quickly when injected into animals. The ex vivo approach avoids this problem altogether," Bennett said.

\section{Recombination frequencies}

An unanswered question is whether the $0.4 \%$ frequency of correction reported in PNAS will be sufficient for clinical benefit.

"From the point of view of therapeutic effect, the recombination frequency is relatively low," said Philip Gregory, VP of research at Sangamo BioSciences Inc.

Sangamo is engineering zinc finger proteins (ZFNs) for a variety of genetic modification functions, including targeted gene modification or correction. The company's lead compound is SB-509, a plasmid encoding a transcription factor that boosts expression of VEGF-A. It is in Phase II testing to treat diabetic neuropathy.

Although the level of genetic correction reported in the paper might not be sufficient to achieve the goal of producing an adequate amount 
of "correct" hemoglobin, Glazer expects that a few modifications to the process could significantly improve recombination levels.

Glazer told SciBX the ultimate goal is to achieve corrected gene levels of about 15-20\%. He thinks modifications to the ex vivo transfection process, as well as repeated infusion of corrected hematopoietic stem cells, will likely overcome the low frequency problem.

"It is plausible to alter the PNA chemical structures to lead to improved activity and increased percentage of transformed genes," said Glazer.

He added that pharmacological modifications that affect gene transfer or improve access to the chromosomes for DNA repair in the cells themselves could improve correction frequency.

Also, there is room for improvement in the process of getting the PNAs and DNA into the cells. "Here, we are using electroporation. Other methods include linking the DNA analogs to very short peptides that translocate through the cell membrane, which could have higher efficacy and boost recombination frequency," said Glazer.

Finally, he told SciBX that "to address the problem of low transformation frequency, a patient could go through repeated transfusion procedures until they get enough stem cells that correct the genetic defect going forward."

\section{Regulation risks}

Some researchers also suggested the PNA technology could raise regulatory red flags.

"From a regulatory perspective, the reason for not pursuing the approach is that the mutations formed by triplex-forming PNAs might cause mutations elsewhere," Bennett cautioned.

"One risk that you do face is cutting the DNA in the wrong places," said Gregory. "The secret to single-locus cleavage is having a long enough recognition site and a technique that only recognizes one point in the genome. The PNA approach is based on Watson-Crick base pairing and uses DNA sequences of only about 9-10 base pairs in length. The pos- sibility of off-target cleavage at unknown locations does exist."

This view was seconded by Carine Giovannangeli, director of research at the biophysics laboratory of the Museum of Natural History in Paris.

"In the paper the target site is 10 base pairs long and might be present in multiple copies in the genome," she said. "Consequently, the length of the target and of the PNA must be increased to guarantee a specific effect and limit off-target effects."

Gregory did say that because the Yale team is focusing on correcting a mutation in an existing gene rather than introducing new genetic material, there should be less regulatory risk. Sangamo has shown that its ZFNs have very low levels of genome-wide cleavage, he added. ${ }^{6}$

Glazer said the PNAs have been patented by Yale and were licensed to Helix Therapeutics LLC, a company he cofounded.

He said Helix would like to raise about $\$ 3$ million to take the PNAs into Phase I testing for one indication, most likely sickle cell disease or thalassemia.

Glazer said the technology is available for nonexclusive licensing.

\section{REFERENCES}

1. Chin, J. et al. Proc. Natl. Acad. Sci. USA; published online Aug. 18 , 2008; doi:10.1073/pnas.0711793105

Contact: Peter M. Glazer, Yale University School of Medicine, New Haven, Conn.

e-mail: peter.glazer@yale.edu

2. Sierakowska, H. et al. Proc. Natl. Acad. Sci. USA 93, 12840-12844 (1996)

3. Olivieri, N. et al. Blood 89, 739-761 (1997)

4. Panigrahi, I \& Marwaha, R. Indian Pediatr. 43, 513-518 (2006)

5. Rogers, F. et al. Proc. Natl. Acad. Sci. USA 99,16695-16700 (2002)

6. Miller, J. et al. Nat. Biotechnol. 25, 778-785 (2007)

\section{COMPANIES AND INSTITUTIONS MENTIONED}

Helix Therapeutics LLC, Cheshire, Conn.

Isis Pharmaceuticals Inc. (NASDAQ:ISIS), Carlsbad, Calif.

Museum of Natural History, Paris, France

Sangamo BioSciences Inc. (NASDAQ:SGMO), Richmond, Calif.

Yale University School of Medicine, New Haven, Conn. 